\title{
The Restorative Effect of Gallic Acid on the Experimental Sciatic Nerve Damage Model
}

\author{
Gokhan Gurkan, ${ }^{1}$ Mumin Alper Erdogan, ${ }^{2}$ Gurkan Yigitturk, ${ }^{3}$ Oytun Erbas ${ }^{4}$ \\ Department of Neurosurgery,' Katip Celebi University Atatürk Training and Research Hospital, Izmir, Turkey \\ Department of Physiology, ${ }^{2}$ Faculty of Medicine, Katip Celebi University, Izmir, Turkey \\ Department of Histology, ${ }^{3}$ Faculty of Medicine, Sitki Kocman University, Mugla, Turkey \\ Department of Physiology, ${ }^{4}$ Istanbul Bilim University Faculty of Medicine, Istanbul, Turkey
}

Objective : Peripheral nerve injuries occur mostly as a result of mechanical trauma. Due to the microvascular deterioration in peripheral nerve damage, it becomes challenging to remove free oxygen radicals. Gallic acid is a powerful antioxidant with anti-inflammatory effects and a free radical scavenger. The purpose of the study is to show that gallic acid contributes to the restorative effect in mechanical nerve damage, considering its antioxidant and anti-inflammatory effects.

Methods : Thirty male Sprague Dawley albino mature rats were included in the study. Ten of them constituted the control group, 10 out of 20 rats for which sciatic nerve damage was caused, constituted the saline group, and 10 formed the gallic acid group. Post-treatment motor functions, histological, immunohistochemical, and biochemical parameters of the rats were evaluated.

Results : Compared to the surgery+saline group, lower compound muscle action potential (CMAP) latency, higher CMAP amplitude, and higher inclined plane test values were found in the surgery+gallic acid group. Similarly, a higher nerve growth factor (NGF) percentage, a higher number of axons, and a lower percentage of fibrosis scores were observed in the surgery+gallic acid group. Finally, lower tissue malondialdehyde (MDA) and higher heat shock protein-70 (HSP-70) values were determined in the surgery+gallic acid group.

Conclusion : Gallic acid positively affects peripheral nerve injury healing due to its anti-inflammatory and antioxidant effects. It has been thought that gallic acid can be used as a supportive treatment in peripheral nerve damage.

Key Words : Gallic acid · Peripheral nerve injuries · Experimental animal models · Sciatic nerve.

\section{INTRODUCTION}

Peripheral nerve damage is a critical reason for morbidity in trauma patients due to the long-term disability it causes ${ }^{7}$. Although peripheral nerve injuries develop for many reasons, they mostly occur due to mechanical trauma". The treatment method adopted to provide post-traumatic nerve integrity is end-to-end repair, especially in short-distance gap injuries. After end-to-end repair, nerve regeneration results are still unsatisfactory regardless of the surgical technique ${ }^{17}$. The complex structure of peripheral nerve cells causes dependence on many factors besides mechanical factors that play a role in

- Received : April 2, 2021 •Revised : May 11, 2021 •Accepted : May 21, 2021

- Address for reprints : Gokhan Gurkan

Department of Neurosurgery, Katip Celebi University Atatürk Training and Research Hospital, Basin Sitesi Mah. Hasan Tahsin Street No: 143, Karabaglar, Izmir 35150, Turkey Tel : +90 50642075 90, Fax : +90 232243 15 30, E-mail : gokhangurkan88@gmail.com, ORCID : https://orcid.org/0000-0003-1839-1014 
nerve healing, such as surgical techniques, in axonal regeneration $^{13)}$.

Gallic acid is an organic acid known as 3,4,5-trihydroxy benzoic acid. This phenolic acid derivative, which has antitumoral and anti-inflammatory effects, is a powerful antioxidant and free radical scavenger. Gallic acid, containing multiple hydroxyl groups, inhibits lipid peroxidase, and it breaks free radical chains by providing multiple protons ${ }^{18)}$. Gallic acid has been shown in experiments to have anti-hyperlipidemic, cardioprotective, and anti-diabetic effects ${ }^{25,27)}$. Additionally, gallic acid can exert neuroprotective effects as a result of its mono- and poly-targeted behavior ${ }^{31,33)}$. Gallic acid also acts as an anti-oxidant in biological organisms ${ }^{23)}$. Furthermore, an acute and subacute toxicity analysis of gallic acid (5000 mg/kg; oral) demonstrated that gallic acid has no significant side effects or organ damage ${ }^{24)}$. As a result of its enhanced safety margins, gallic acid could be approved for use in chronic neurodegenerative diseases. Nevertheless, academic evidence supporting the use of gallic acid in sciatic nerve damage is insufficient. It is crucial to develop new treatment strategies to improve and significantly accelerate regeneration in mechanical nerve damage. The study aims to show that gallic acid contributes to the restorative effect in mechanical nerve damage, considering its antioxidant and anti-inflammatory effects, by immunohistochemical, electrophysiological, and motor function tests.

\section{MATERIALS AND METHODS}

The experimental procedures employed in the present study were approved by the Animal Ethics Committee (2011-176/b).

\section{Animals}

Thirty male Sprague Dawley albino mature rats weighing 200-220 g were used in this study. Animals were fed ad libitum and housed in pairs in steel cages having a temperaturecontrolled environment $\left(22 \pm 2^{\circ} \mathrm{C}\right)$ with 12 -hour light/dark cycles.

The rats used in the experiment were obtained from the experimental animal laboratory. All experiments were performed according to the Guide for the Care and Use of Laboratory Animals, as confirmed by the National Institutes of Health (USA).

\section{Chemicals and drugs}

Gallic acid (purity $\geq 98 \%$; Sigma Chemical Co., St Louis, MO, USA), Ketamine and Xylazine (Alfasan International B.V. Holland, Holland, The Netherlands), heat shock protein-70 (HSP-70) enzyme-linked immunosorbent assay (ELISA) kit (USCN; Life Science Inc., Wuhan, China), and NGF antibody (Santacruz Biotechnology, Santa Cruz, CA, USA) were used. All other chemicals for various analyses were purchased either from Sigma Chemical Co.

\section{Experimental protocol}

Thirty male Sprague Dawley rats were included in the study. Twenty rats were considered as experimental groups, and surgical sciatic nerve dissection and repair operation was performed. Ten rats formed a control group $(n=10)$, and no surgical operation or drug treatment was applied. The experimental group (20 rats) was divided into two groups. Surgery+saline group $(n=10)$ rats were assigned to a placebo group and were given 1 $\mathrm{mL} / \mathrm{kg} /$ day $0.9 \% \mathrm{NaCl}$ saline intraperitoneally (i.p.) following the surgery. Surgery+gallic acid group $(n=10)$ rats were given 20 $\mathrm{mg} / \mathrm{kg} /$ day gallic acid i.p., following the surgery. All medications were administered for 12 weeks. The motor function test was performed at the end of 12 weeks. Electromyography (EMG) recordings were done after the motor function test. Finally, blood samples were taken by tail vein puncture for biochemical analysis, and then the animals were euthanized, and sciatic nerve samples were taken for immunohistochemistry analysis.

\section{Surgical procedure}

Under the induction of general anesthesia of $75 \mathrm{mg} / \mathrm{kg}$ ketamine (Alfamine; Alfasan International B.V. Holland) and 10 g/kg xylazine (Alfazyne; Alfasan International B.V. Holland) administered by intraperitoneal injection, rats were fixed to the operating table in the prone position. Both sciatic nerves were exposed from $1 \mathrm{~cm}$ distal of the sciatic notch to $1 \mathrm{~cm}$ distal to trifurcation of the nerve using an aseptic technique. $3-3.5 \mathrm{~cm}$ long nerve segments above the trifurcation were dissected carefully to isolate the sciatic nerve from surrounding soft tissue. The nerves were then transected by the micro scissors at a level of $1.5 \mathrm{~cm}$ above the trifurcation (i.e., starting point of the tibial nerve, common peroneal nerve, and caudal sural cutaneous nerve). Nerves were repaired with three epineural sutures (Ethilon ${ }^{\circledR} 9-0$; Ethicon, Somerville, NJ, USA) by the same surgeon. 
The wound was closed with a Vicryl ${ }^{\circledR} 3-0$ (Ethicon), and the rats were allowed to recover. After the recovery from anesthesia, rats were put back to their cages and allowed freely to get food and water.

\section{Assessment of motor function}

The inclined-plate test evaluated the rats' motor performances according to the method described by Tator and Fehlin$\mathrm{gs}^{30)}$. Briefly, the rat was placed oblique to the long axis of an inclined plate. The initial angle of the inclined plate was $10 \mathrm{de}-$ grees. The incline angle slowly increased, and the plate's maximum angle on which the rat preserved its position for $5 \mathrm{sec}-$ onds without falling was recorded as a motor score. The inclined plate angle was measured three times in each rat to find an average value.

\section{Electrophysiological recordings}

Rats were anesthetized by a combination of ketamine hydrochloride at a dose of $80 \mathrm{mg} / \mathrm{kg}$ (Alfamine; Alfasan International B.V. Holland) and $10 \mathrm{mg} / \mathrm{kg}$ of xylazine hydrochloride (Alfazyne; Alfasan International B.V. Holland). Electrophysiological recordings (EMG studies) were performed in all groups at the end of the study. EMG was obtained three times from both sciatic nerves stimulated supramaximal (intensity $10 \mathrm{~V}$, duration $0.05 \mathrm{~ms}$, frequency $1 \mathrm{~Hz}$, in the range of 0.5-5000 $\mathrm{Hz}, 40 \mathrm{kHz} / \mathrm{s}$ with a sampling rate) by a bipolar subcutaneous needle stimulation electrode (BIOPAC Systems, Inc., Santa Barbara, CA, USA) from the sciatic notch. Compound muscle action potential (CMAP) was recorded from 2-3 interosseous muscles through unipolar platinum electrodes. Data were evaluated using Biopac Student Lab Pro version 3.6.7 software (BIOPAC Systems, Inc.) with distal latency and amplitude of CMAP as the parameters. During the EMG recordings, the rectal temperatures of the rats were monitored by a rectal probe (HP Viridia 24-C; Hewlett-Packard Company, Palo Alto, CA, USA), and the temperature of each rat was kept at approximately $36-37^{\circ} \mathrm{C}$ by a heating pad. All experiments were performed between 10:00 a.m. and 02:00 p.m.

\section{Biochemical analysis of sciatic nerve tissue}

After decapitation, sciatic nerves were rapidly removed and stored at $-20^{\circ} \mathrm{C}$ until biochemical analysis. For tissue analysis, whole nerve tissues were homogenized with a glass homogenizer in 5 volumes of phosphate-buffered saline $(\mathrm{pH}, 7.4)$ and centrifuged at $5000 \mathrm{~g}$ for 15 minutes. The supernatant was then collected, and total protein concentration in the nerve homogenates was determined according to Bradford's method using bovine serum albumin as the standard ${ }^{6}$.

The sciatic levels of HSP-70 in the tissue supernatants were measured using commercially available rat ELISA kits. All samples from each animal were measured in duplicate according to the manufacturer's guidelines.

\section{Measurement of nerve lipid peroxidation}

Lipid peroxidation was determined in tissue samples by measuring malondialdehyde (MDA) levels as thiobarbituric acid reactive substances. Briefly, trichloroacetic acid and trichloroacetic acid and thiobarbituric acid reactive substance reagent were added to the tissue samples, then mixed and incubated at $100^{\circ} \mathrm{C}$ for 60 minutes. After cooling on ice, the samples were centrifuged at $3000 \mathrm{rpm}$ for 20 minutes, and the absorbance of the supernatant was read at $535 \mathrm{~nm}$. MDA levels were calculated from the standard calibration curve using tetra ethoxy propane and expressed as nmol/ $\mu \mathrm{g}$ protein.

\section{Histology and quantitative immunohistochemis- try}

Rats were perfused intracardially with $4 \%$ formaldehyde for histology and quantitative immunohistochemistry. Briefly, sciatic nerves were embedded in paraffin, sectioned at $5 \mu \mathrm{m}$ thickness via microtome (Leica RM 2145; Leica Instruments GmbH, Nussloch, Germany), and stained with hematoxylin-eosin (H\&E). Axons were detected through H\&E staining. The sciatic epineurium nerve's thickness was measured, and the stained tissue sections were then examined with an Olympus C-5050 digital camera (Olympus, Tokyo, Japan) mounted on an Olympus BX51 microscope (Olympus). In each group, the Image-Pro Express 4.5 (Media Cybernetics, Inc., Rockville, MD, USA) program was used to measure the total axon number, the thicknesses of the perineural layers in the middle regions of the grafts, and the level of fibrosis covering these layers in the histological specimens and then analyzed statistically.

For immunohistochemical examination, sections were incubated with $\mathrm{H}_{2} \mathrm{O}_{2}(10 \%)$ for 30 minutes. to eliminate endogenous peroxidase activity and then blocked with normal goat serum (Invitrogen, Carlsbad, CA, USA) for 1 hour at room temperature. Subsequently, sections were incubated with primary antibodies (1/100; Santacruz Biotechnology) against 
nerve growth factor (NGF) for 24 hours at $4^{\circ} \mathrm{C}$. Antibody detection was carried out by the Histostain-Plus Bulk kit (Invitrogen) against rabbit immunoglobulin G, and 3,3' diaminobenzidine was used to visualize the final product. All sections were washed in phosphate-buffered saline, examined under an Olympus BX51 microscope (Olympus), and photographed by Olympus C-5050 digital camera (Olympus). All groups and six sections from each animal were used for quantitative immunohistochemistry. Two blinded observers counted the total immune-positive Schwann cells and the number of axons under a light microscope at $\times 10$ and $\times 20$ magnification. Data were expressed as the mean \pm standard error of the mean (SEM).

\section{Statistical analysis}

SPSS version 20.0 (IBM Corp., Armonk, NY, USA) was used to perform statistical analysis. All data were evaluated by oneway analysis of variance. Post-hoc Tukey's honestly significant difference test was used for post hoc multiple comparisons. Also, the groups of nonparametric variables were compared using the Mann-Whitney U test. In addition, the ShapiroWilk test was used for parametric-nonparametric differentiation. Results are presented as mean+SEM. $p<0.05$ was accepted as statistically significant.

\section{RESULTS}

\section{Inclined plane test/motor function results}

At the end of the study, motor functions were evaluated by using the inclined plane test. A statistically significant decrease of inclined plane degrees was observed in the surgery+saline

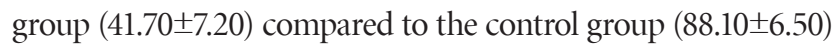
$(p<0.001)$. The rats in the surgery+gallic acid group were able to climb significantly higher degrees $(65.80 \pm 8.40)$ in the inclined plane compared to the surgery+saline group (41.70 \pm 7.20$)$ $(p<0.001)$ (Table 1).

\section{Evaluation of electrophysiological recordings}

The amplitudes of CMAP decreased significantly in the surgery+saline group $(2.50 \pm 0.31)$ compared to the control group (11.53 \pm 2.04$)(p<0.001)$. When compared to the control group (2.31 \pm 0.19$)$, the latency of CMAP was significantly extended in the surgery+saline group $(3.69 \pm 0.44)(p<0.05)$.

Even then, the amplitudes of CMAP were significantly greater in the surgery+gallic acid group $(6.72 \pm 1.13)$ than in the surgery+saline group $(2.50 \pm 0.31)(p<0.001)$. CMAP latency was significantly reduced in the surgery+gallic acid group $(2.90 \pm 0.19)$ over the surgery+saline group $(3.69 \pm 0.44)$ $(p<0.001)$ (Fig. 1 and Table 1).

Table 1. Intergroup comparison of motor functions and EMG values

\begin{tabular}{lccc}
\hline & Control group & Surgery+saline group & Surgery+gallic acid group \\
\hline EMG CMAP latency (MS) & $2.31 \pm 0.19$ & $3.69 \pm 0.44^{*}$ & $2.90 \pm 0.19^{\ddagger}$ \\
EMG CMAP amplitude $(\mathrm{mV})$ & $11.53 \pm 2.04$ & $2.50 \pm 0.31^{\dagger}$ & $6.72 \pm 1.13^{\S}$ \\
Inclined plane score $\left(^{\circ}\right)$ & $88.10 \pm 6.50$ & $41.70 \pm 7.20^{\dagger}$ & $65.80 \pm 8.40^{\S}$ \\
\hline
\end{tabular}

Values are presented as mean \pm standard error of the mean. ${ }^{*} p<0.05,{ }^{\dagger} p<0.001$; surgery+saline group compared with control group. ${ }^{\ddagger} p<0.001,{ }^{\S} p<0.001$; surgery+gallic acid group compared with surgery+saline group. EMG : electromyography, CMAP : compound muscle action potential

(A)

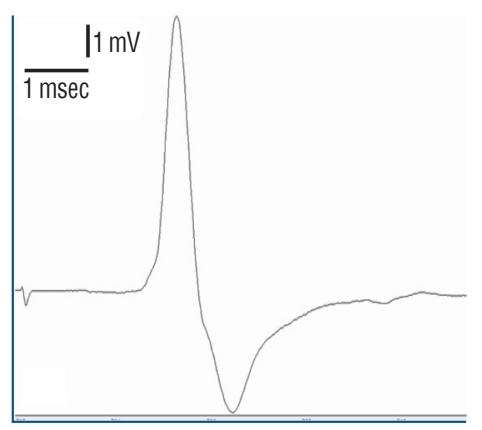

(B)

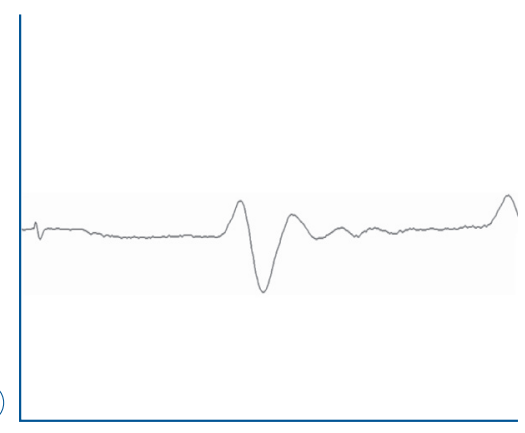

(C)

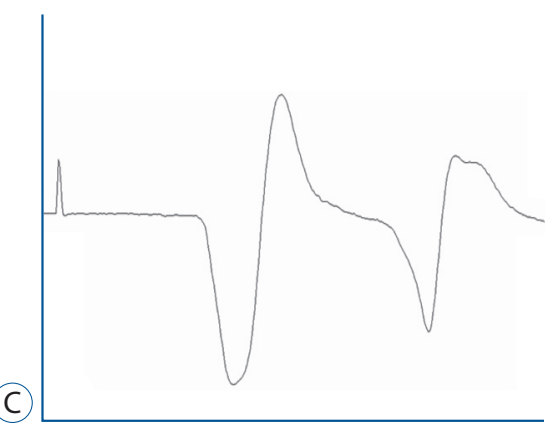

Fig. 1. Electromyography of (A) control group, (B) surgery+saline group, and (C) surgery+gallic acid group. 


\section{Histology and quantitative immunohistochemis- try}

At the conclusion of the experiments, Wallerian degeneration of the sciatic nerves led to a significant decrease in the number of axons in the surgery+saline group (165.40 \pm 45.90$)$ when compared to the control group (1318.00土56.10) $(p<0.001)$, but not in the surgery+gallic acid group. The surgery+gallic acid group (408.20 \pm 34.50$)$ had a significantly greater number of axons than the surgery+saline group

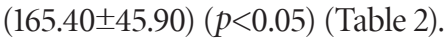

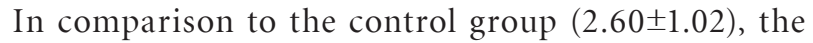
surgery+saline group's fibrosis score $(88.10 \pm 10.20)$ in sciatic nerve tissues increased significantly $(p<0.001)$ (Table 2 and

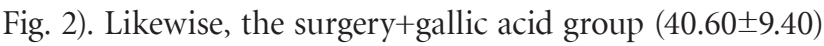

had a higher fibrosis score than the control group (2.60 \pm 1.02$)$. This increase, though, was significantly lower than that observed in the surgery+saline group $(88.10 \pm 10.20)(p<0.001)$.

When compared to the control group (54.20 \pm 8.50$)$, the surgery+saline group's NGF immuno-expression in Schwann cells $(2.40 \pm 0.80)$ was significantly lower $(p<0.05)$. In comparison to the surgery+saline group $(2.40 \pm 0.80)$, NGF immunoexpression in the Schwann cells increased significantly in the surgery+gallic acid group $(14.70 \pm 2.30)(p<0.05)$ (Table 2 and Fig. 2).

\section{Evaluation of lipid peroxidation and oxidative stress}

When compared to the control group (116.70 \pm 10.10$)$, the

Table 2. Intergroup comparison of histological and immunohistochemical evaluation

\begin{tabular}{lccc}
\hline & Control group & Surgery+saline group & Surgery+gallic acid group \\
\hline NGF expression on Schwann cell (\%) & $54.20 \pm 8.50$ & $2.40 \pm 0.80^{*}$ & $14.70 \pm 2.30^{\ddagger}$ \\
Total axon number & $1318.00 \pm 56.10$ & $165.40 \pm 45.90^{\dagger}$ & $408.20 \pm 34.50^{\ddagger}$ \\
Fibrosis score (\%) & $2.60 \pm 1.02$ & $88.10 \pm 10.20^{\dagger}$ & $40.60 \pm 9.40^{\S}$ \\
\hline
\end{tabular}

Values are presented as mean \pm standard error of the mean. ${ }^{*} p<0.05,{ }^{\dagger} p<0.001$; surgery+saline group compared with control group. ${ }^{\ddagger} p<0.05,{ }^{\S} p<0.001$; surgery+gallic acid group compared with surgery+saline group. NGF : nerve growth factor
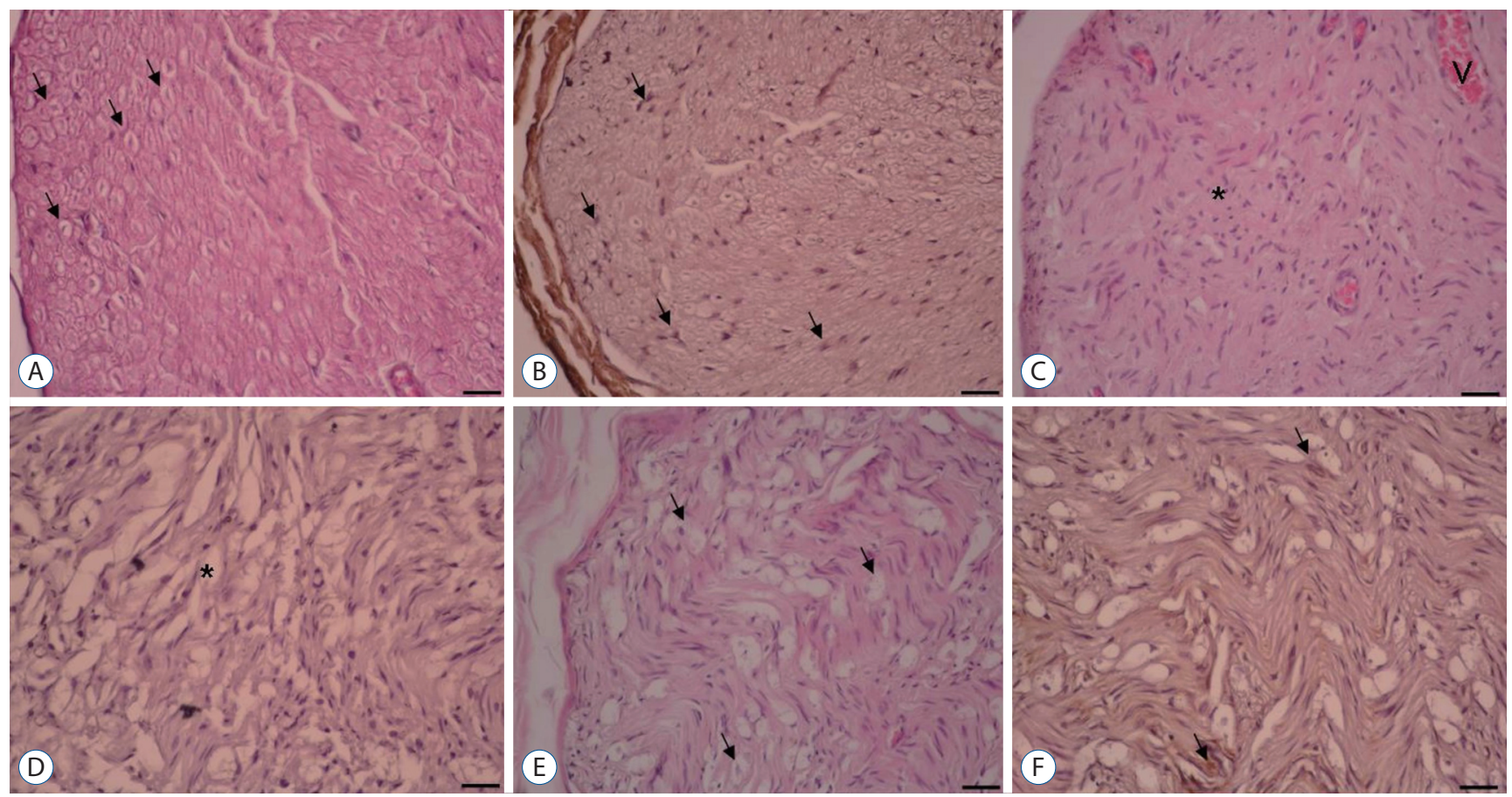

Fig. 2. $\times 20$ Magnification. Hematoxylin \& Eosin and NGF immunostaining. A and B : Control group. Normal axon and Schwann cell (arrows). C and D : Surgery+saline group. Increased fibrosis (asterisk) was shown. Very diminished axon, Schwann cell, and NGF immunoexpression (asterisk). E and F : Surgery+gallic acid group. Increased axon, Schwann cell, and NGF immunoexpression (arrows). NGF : nerve growth factor. 
Table 3. Intergroup comparison of biochemical evaluation

\begin{tabular}{lccc} 
& Control group & Surgery+saline group & Surgery+gallic acid group \\
\hline Tissue MDA (nmol/ $\mathrm{mg})$ & $116.70 \pm 10.10$ & $303.80 \pm 16.50^{*}$ & $203.50 \pm 14.30^{\dagger}$ \\
Tissue HSP-70 (mcg/mg protein) & $10.60 \pm 2.08$ & $14.50 \pm 3.60$ & $32.90 \pm 7.50^{\dagger}$ \\
\hline
\end{tabular}

${ }^{*} p<0.001$; surgery+saline group compared with control group. ${ }^{\dagger} p<0.001$; surgery+gallic acid group compared with surgery+saline group. MDA : malondialdehyde, HSP-70 : heat shock protein-70

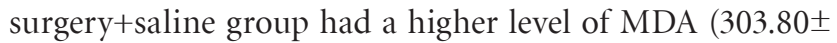
16.50), which is a marker of oxidative stress $(p<0.001)$. MDA levels were significantly lower in the surgery+gallic acid group $(203.50 \pm 14.30)$ than in the surgery+saline group $(303.80 \pm$ 16.50) $(p<0.001)$ (Table 3).

\section{Evaluation of HSP-70 as an inflammatory marker}

HSP-70 proteins have been linked to the regulation of immune responses and the modulation of inflammation by a variety of mechanisms. Normally, HSP-70 can bind to endocytic receptors and be endocytosed, acquiring access to antigen presentation routes and modifying the cell phenotype toward one that is tolerogenic, resulting in the development of the anti-inflammatory cytokine interleukin (IL)-10 and, subsequently, immunosuppression. HSP-70 inhibits tumor necrosis factor (TNF)- $\alpha$ development in DCs by downregulating CD86 and Major Histocompatibility Complex class II expression. Regarding this information, we evaluated the HSP-70 levels in our groups. Both groups showed an increase in tissue HSP-70 levels as compared to the control group (10.60土2.08), while

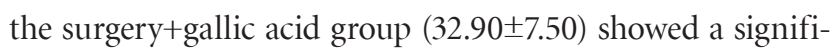
cant increase as compared to the surgery+saline group (14.50 $\pm 3.60)(p<0.001)$ (Table 3).

\section{DISCUSSION}

Neural tissue and microvascular structure deteriorate following mechanical impairment in peripheral nerve damage. Microcirculation is impaired after endothelial damage, and free oxygen radicals begin to be released. Microcirculation disintegration and a decrease in antioxidant enzymes make it challenging to remove free oxygen radicals. This condition causes a delay in providing the appropriate environment in the nerve healing process ${ }^{3,8)}$.

In experimental studies on sciatic nerve damage, various antioxidant and anti-inflammatory supportive therapies have been examined comparatively. The neuroprotective effects of these substances on both mechanical damage and ischemiareperfusion injury were evaluated ${ }^{21,22,26)}$. Gallic acid is one of the treatments showing these effects.

The anti-inflammatory efficacy of gallic acid is created by suppressing pro-inflammatory cytokines and chemokines ${ }^{20)}$. This efficacy has previously been demonstrated in experimental animal models on organs such as the lung and stomach ${ }^{2,29)}$. The effectiveness of gallic acid in peripheral nerve injury healing was evaluated only by Hajimoradi et al. ${ }^{11)}$ in the literature.

In our study, the inclined plane test evaluated the motor functions of test animals. Accordingly, the surgery+gallic acid group results were found to be higher than in the surgery+saline group. Electrophysiological parameters during nerve recovery were assessed using EMG. Similarly, compared to the surgery+saline group, CMAP latency was lower, and CMAP amplitude was higher in the surgery+gallic acid group.

NGF is a mediator that plays a vital role in the development and regeneration of the peripheral nervous system ${ }^{12)}$. It contributes to axonal extension and myelinization in Schwann cells $^{10)}$. In our study, fibrosis score percentages, axon numbers, and NGF percentage parameters in Schwann cells were examined in histological and immunohistochemical evaluations to observe nerve healing. As a result of the evaluation, lower fibrosis score percentage, higher axon counts, and higher NGF percentage favored better recovery in the surgery+gallic acid group.

Lipid peroxidation is a critical marker indicating oxidative stress caused by free oxygen molecules. MDA indicates the presence of lipid peroxidation, and an increase in MDA levels means increased oxidative stress and deterioration in the microenvironment ${ }^{16)}$. Additionally, gallic acid was discovered to be an adaptable scavenger, capable of effectively inhibiting a wide range of reactive oxygen and nitrogen species through electron transfer ${ }^{19)}$. When natural products containing gallic acid were given to laboratory animals, antioxidant effects were observed in various tissues, including the lungs and stomach, 
according to the literature ${ }^{1,28)}$. Gallic acid has also been shown to have anti-inflammatory properties by inhibiting the development of pro-inflammatory cytokines and chemokines ${ }^{4}$. According to another study, gallic acid significantly healed MDA and superoxide dismutase levels but had little impact on the catalase amounts in diabetic rat testes. With the same anti-inflammatory pathways, it drastically reduced TNF- $\alpha$, nitric oxide synthase-2, and vascular endothelial growth factor levels ${ }^{34)}$. The treatment of gallic acid has ameliorative effects against TNF- $\alpha$ in paclitaxel-induced neuropathic pain in mice model ${ }^{15)}$. HSP-70 is in the heat shock protein family and has cell protective efficacy ${ }^{14}$. Studies revealed that HSP-70 prevents cell death in damaged neurons are available in the literature $^{32}$. HSP treatment has been shown to inhibit or arrest inflammatory damage in laboratory disease models, and in early clinical trials in patients with chronic inflammatory disorders, HSP proteins and peptides have been shown to stimulate the development of anti-inflammatory cytokines, suggesting that HSP possesses immunoregulatory capacity. Thus, the involvement of immune responses to HSP in inflammatory diseases can be interpreted as the immune system attempting to fix the inflammatory state ${ }^{5}$. As we mentioned before, HSP-70 proteins have been linked to the regulation of immune responses and the modulation of inflammation by a variety of mechanisms. Normally, HSP-70 can bind to endocytic receptors and be endocytosed, acquiring access to antigen presentation routes and modifying the cell phenotype toward one that is tolerogenic, resulting in the development of the anti-inflammatory cytokine IL-10 and, subsequently, immunosuppression. HSP-70 inhibits TNF- $\alpha$ development in DCs by downregulating CD86 and MHC class II expression ${ }^{5}$. For all these reasons, tissue MDA and HSP-70 levels were compared in our study. Accordingly, statistically significant lower MDA levels and higher HSP-70 levels were found in the surgery+gallic acid group compared to the surgery+saline group. All these results showed that gallic acid had a more beneficial effect on recovery than placebo.

There is a limited number of studies in the literature investigating the effect of gallic acid on sciatic nerve damage with our study parameters. Kaur and Muthuraman ${ }^{15)}$ showed that gallic acid could ameliorate paclitaxel-induced neuropathic pain in mice model. However, this was a neuropathic pain model, and in our study, we focused on the ameliorative effects of gallic acid on motor functions in the sciatic nerve dama- ge model. Therefore, it was challenged to compare our study findings with the literature. This situation is also one of the limitations of our study. Another limitation is that since our study is an experimental animal study, the number of animals we used could be high due to ethical concerns. Further studies involving more subjects and comparing gallic acid with other accepted treatments are needed.

\section{CONCLUSION}

In conclusion, we demonstrated that gallic acid positively affects peripheral nerve injury healing due to its anti-oxidant and anti-inflammatory effects. A significant enhancement in healing was observed in the surgery+gallic acid group compared to the placebo group. Regarding our findings, it has been thought that gallic acid may be used as a supportive treatment for peripheral nerve damages. More comparative studies are also needed.

\section{CONFLICTS OF INTEREST}

No potential conflict of interest relevant to this article was reported.

\section{INFORMED CONSENT}

This type of study does not require informed consent.

\section{AUTHOR CONTRIBUTIONS}

\author{
Conceptualization : GG, MAE, OE \\ Data curation : GG, MAE, GY \\ Formal analysis: GG, MAE, OE \\ Funding acquisition : $\mathrm{OE}$ \\ Methodology: GG, MAE, OE \\ Project administration : $\mathrm{OE}$ \\ Visualization : GY, OE \\ Writing - original draft : GG \\ Writing - review \& editing: GG, MAE
}




\section{ORCID}

\begin{tabular}{|c|c|}
\hline Gokhan Gurkan & https://orcid.org/0000-0003-1839-1014 \\
\hline Mumin Alper Erdogan & rg/0000-0003-0048-444X \\
\hline Gurkan Yigitturk & rg/0000-0002-5315-253X \\
\hline Dytun 1 & cid.org/0000-0002-2515-2946 \\
\hline
\end{tabular}

\section{References}

1. Arora P, Ansari SH, Anjum V, Mathur R, Ahmad S : Investigation of antiasthmatic potential of Kanakasava in ovalbumin-induced bronchial asthma and airway inflammation in rats. J Ethnopharmacol 197 : 242-249, 2017

2. Arora P, Ansari SH, Najmi AK, Anjum V, Ahmad S : Investigation of antiasthmatic potential of dried fruits of Vitis vinifera $\mathrm{L}$. in animal model of bronchial asthma. Allergy Asthma Clin Immunol 17 : 12, 2016

3. Bagdatoglu C, Saray A, Surucu HS, Ozturk H, Tamer L : Effect of trapidil in ischemia/reperfusion injury of peripheral nerves. Neurosurgery 51 : 212-219; discussion 219-220, 2002

4. Bolzani V, Soares $C$, Pezzuto J, Luqman S, Morais M, Kondratyuk T, et al. : Suppression of TNF- $\alpha$ induced NFKB activity by gallic acid and its semi-synthetic alkyl-gallates: possible role in cancer chemoprevention. Nature Precedings, 2012 [Epub ahead of print]

5. Borges TJ, Wieten L, van Herwijnen MJ, Broere F, Van Der Zee R, Bonorino $C$, et al. : The anti-inflammatory mechanisms of Hsp70. Front Immunol 3 : 95, 2012

6. Bradford MM : A rapid and sensitive method for the quantitation of microgram quantities of protein utilizing the principle of protein-dye binding. Anal Biochem $72:$ 248-254, 1976

7. Chen $M B$, Zhang $F$, Lineaweaver $W C$ : Luminal fillers in nerve conduits for peripheral nerve repair. Ann Plast Surg 57 : 462-471, 2006

8. Fairbairn NG, Meppelink AM, Ng-Glazier J, Randolph MA, Winograd JM : Augmenting peripheral nerve regeneration using stem cells: a review of current opinion. World J Stem Cells 7 : 11-26, 2015

9. Faroni A, Mobasseri SA, Kingham PJ, Reid AJ : Peripheral nerve regeneration: experimental strategies and future perspectives. Adv Drug Deliv Rev 82-83 : 160-167, 2015

10. Gao C, Ma S, Ji Y, Wang JE, Li J : Siatic nerve regeneration in rats stimulated by fibrin glue containing nerve growth factor: an experimental study. Injury 39 : 1414-1420, 2008

11. Hajimoradi M, Fazilati M, Gharib-Naseri MK, Sarkaki A : Gallic acid and exercise training improve motor function, nerve conduction velocity but not pain sense reflex after experimental sciatic nerve crush in male rats.

Avicenna J Phytomed 5 : 288-297, 2015

12. Henderson CE : Role of neurotrophic factors in neuronal development. Curr Opin Neurobiol 6 : 64-70, 1996

13. Huang W, Begum R, Barber T, Ibba V, Tee NC, Hussain M, et al. : Regenerative potential of silk conduits in repair of peripheral nerve injury in adult rats. Biomaterials $33:$ 59-71, 2012

14. Kalmar B, Burnstock G, Vrbová G, Urbanics R, Csermely P, Greensmith $L$ : Upregulation of heat shock proteins rescues motoneurones from axotomy-induced cell death in neonatal rats. Exp Neurol 176 : 87-97, 2002

15. Kaur S, Muthuraman A : Ameliorative effect of gallic acid in paclitaxelinduced neuropathic pain in mice. Toxicol Rep 6 : 505-513, 2019

16. Koracevic D, Koracevic G, Djordjevic V, Andrejevic S, Cosic V : Method for the measurement of antioxidant activity in human fluids. J Clin Pathol 54 : 356-361, 2001

17. Lundborg G : Enhancing posttraumatic nerve regeneration. J Peripher Nerv Syst 7 : 139-140, 2002

18. Ma J, Luo XD, Protiva P, Yang H, Ma C, Basile MJ, et al. : Bioactive novel polyphenols from the fruit of Manilkara zapota (Sapodilla). J Nat Prod 66 : 983-986, 2003

19. Marino T, Galano A, Russo N : Radical scavenging ability of gallic acid toward $\mathrm{OH}$ and $\mathrm{OOH}$ radicals. Reaction mechanism and rate constants from the density functional theory. J Phys Chem B 118 : 10380-10389, 2014

20. Morais MC, Luqman S, Kondratyuk TP, Petronio MS, Regasini LO, Silva $\mathrm{DH}$, et al. : Suppression of TNF- $\alpha$ induced NFKB activity by gallic acid and its semi-synthetic esters: possible role in cancer chemoprevention. Nat Prod Res 24 : 1758-1765, 2010

21. Ogut E, Yildirim FB, Sarikcioglu L, Aydin MA, Demir N : Neuroprotective effects of ozone therapy after sciatic nerve cut injury. Kurume Med J 65 : 137-144, 2020

22. Ozyigit F, Kucuk A, Akcer S, Tosun M, Kocak FE, Kocak C, et al. : Different dose-dependent effects of ebselen in sciatic nerve ischemiareperfusion injury in rats. Bosn J Basic Med Sci 15 : 36-43, 2015

23. Park HH, Ko SC, Oh GW, Jang YM, Kim YM, Park WS, et al. : Characterization and biological activity of PVA hydrogel containing chitooligosaccharides conjugated with gallic acid. Carbohydr Polym 198 : 197205, 2018

24. Rajalakshmi K, Devaraj H, Niranjali Devaraj S : Assessment of the noobserved-adverse-effect level (NOAEL) of gallic acid in mice. Food Chem Toxicol 39 : 919-922, 2001

25. Sanlier N, Atik I, Atik A : A minireview of effects of white tea consumption on diseases. Trends Food Sci Technol 82 : 82-88, 2018

26. Shokouhi G, Tubbs RS, Shoja MM, Hadidchi S, Ghorbanihaghjo A, Roshangar $\mathrm{L}$, et al. : Neuroprotective effects of high-dose vs low-dose melatonin after blunt sciatic nerve injury. Childs Nerv Syst 24 : 111117,2008

27. Singh J, Saha L, Singh N, Kumari $P$, Bhatia A, Chakrabarti A : Study of nuclear factor-2 erythroid related factor-2 activator, berberine, in paclitaxel induced peripheral neuropathy pain model in rats. J Pharm Pharmacol 71 : 797-805, 2019

28. Tamboli FA, More HN : Evaluation of antiulcer and antioxidant activity of Barleria gibsoniDalz. leaves. Pharmacognosy Res 8 : 226-230, 2016

29. Tamboli FA, More HN : Anthelmintic activity of leaves extract of Barleria gibsoni Dalz. against Pheretima posthuma. JPP 5 : 250-252, 2016

30. Tator $\mathrm{CH}$, Fehlings MG : Review of the secondary injury theory of acute 
Gallic Acid on Sciatic Nerve Damage | Gurkan G, et al.

spinal cord trauma with emphasis on vascular mechanisms. J Neurosurg $75: 15-26,1991$

31. Teixeira J, Oliveira C, Cagide F, Amorim R, Garrido J, Borges F, et al. : Discovery of a new mitochondria permeability transition pore (mPTP) inhibitor based on gallic acid. J Enzyme Inhib Med Chem 33 : 567576, 2018

32. Tidwell $\mathrm{L}$, Houenou LJ, Tytell $M$ : Administration of Hsp70 in vivo inhibits motor and sensory neuron degeneration. Cell Stress Chaperones
$9: 88-98,2004$

33. Verma S, Singh A, Mishra A : Gallic acid: molecular rival of cancer. Environ Toxicol Pharmacol 35 : 473-485, 2013

34. Yigitturk G, Acara AC, Erbas O, Oltulu F, Yavasoglu NUK, Uysal A, et al. : The antioxidant role of agomelatine and gallic acid on oxidative stress in STZ induced type I diabetic rat testes. Biomed Pharmacother 87 : 240-246, 2017 